\title{
A Conceptual Model for the Identification of Suitable Personnel to Operate in High-risk, Physically Demanding Environments
}

\author{
R.A. Mouthaan, S. El Sawah and M.J. Ryan \\ Capability Systems Centre, School of Engineering and IT (SEIT), The University of New South Wales at The \\ Australian Defence Force Academy, Australian Capital Territory \\ Email:r.mouthaan@adfa.edu.au
}

\begin{abstract}
When determining the suitability of human resources for employment in high-risk, physically demanding environments, it is crucial to ensure the most appropriate person is selected for each role. While the concepts and practices are well established (i.e. what they need to do, how they need to do it, and the desired effects while they are doing it) there is little research into who should be doing it. This gap may have significant effects on the task outcomes, the individual performing the task, and ultimately how well resources are managed. To bridge this gap, there is a need for assessment tools that can make use of basic fitness and medical assessments to determine an individual's predisposition to conduct a specific role in harsh environments. Using statistical modelling techniques - specifically Bayesian Networks (BN) - this research develops a conceptual model for identifying the suitability of personnel to operate in high-risk, physically demanding environments.
\end{abstract}

The aim of this ongoing research is to make use of the BN modelling methodology to help generate knowledge and tools that support the selection of personnel performing high risk physical roles. BN modelling offers several advantages, such as the ability to integrate qualitative and quantitative data, ease of communication due to transparent model structure, as well as the ability to handle data uncertainty. BN is also an adaptive modelling technique, which means that the model structure and parameters can be updated as new data becomes available. The intended outcome of the $\mathrm{BN}$ is to develop an integrated understanding of the factors (i.e. individual, task and environment), while illuminating knowledge gaps and further areas that warrant investigation.

We use a case study approach to support the development and validation of the BN. The selected case study aims to develop an assessment tool to support the selection of individuals to operate in individual protective equipment (IPE) in a chemical, biological, radiological, nuclear or explosive (CBRNE) environment. Here the intended outcome is to develop a metric for selecting the most appropriate personnel to operate within a CBRNE environment. This case study is a topical showcase as it will support a gap in the ability to select CBRNE operators to support military services to respond and operate at times where there are increasing threats of terrorist attacks. Findings from the study can be transferred to other applications, such as the selection of personnel to work in IPE for first responders, emergency and medical services.

Keywords: Selection criteria, Bayesian Network (BN), high risk environments 


\section{INTRODUCTION}

The impacts of terrorism have become a staple of day to day life, creating a world climate where both state and non-state actors are seeking more efficient ways to maximise weapon effectiveness, both physically and psychologically. Any chemical, biological, radiological, nuclear or explosive (CBRNE) weapons provide a range of solutions that achieve this, within a relatively low budget and with devastating consequences. It may only be a matter of time before these actors attain and can deploy CBRNE weapons. (https://www.businessinsider.com.au/global-terrorist-attacks-past-20-years-in-maps-2017 accessed 15 Jul 17)

The recognition of the importance of the human as an element of the greater system of resources highlights the requirement to maximize the effectiveness of that resource. If selected appropriately, the whole system will introduce efficiencies and therefore costs savings for the employment and management of the human resource (Arthur, 1994). The critical requirement for human resources that can operate effectively in highrisk, physically demanding environments highlights the need to select the most appropriate person for the role. To combat this, coalition nations need to improve the preparedness of defence, emergency and medical services to mitigate, fight, defend, operate, escape, recover and recuperate in high risk, physically demanding environments. Key to this is the selection of the appropriate personnel to operate in the environment or, at least, to have an awareness of an individual's capacity to operate in that environment.

The aim of this study is to begin developing an assessment tool that can use the results from basic fitness and medical assessments to determine the suitability of personnel for employment in specific environments. This research achieves this be developing a conceptual model (CM) for identifying the suitability of personnel to operate in high-risk, physically demanding environments then uses Bayesian Networks (BN) to develop a tool to assess this. While the concepts and practices are well established (i.e. what they need to do, how they need to do it, and the desired effects while they are doing it) there is little work done on who should be doing it. Initial research indicates that there is not currently a means of using the results of basic fitness and medical assessments to determine an individual's predisposition to operating in high-risk, physically demanding environments (Bartel, 1994 and Becker and Gerhart, 1996). Consequently, readily available periodic medical and physical assessment results are not utilised to select personnel to operate in these environments.

The wider research study is organised into five phases; Problem Framing, Model Conceptualisation, Model Development, Model Validation and Model Use. This paper addresses Problem Framing, Model Conceptualisation and Model Development.

Bayesian Networks. A Bayesian network (BN) is a model used to create a sequential approach to the complexities of probability distribution. To accommodate the case where our understanding on what is going on is incomplete, the model describes things probabilistically and provides the opportunity to utilise other means to fill knowledge gaps (Charniak 1991, Hart and Pollino 2008, Hart and Pollino 2009, Wooldidge and Done 2003). BN are often considered suitable for modelling environmental systems due to their ability to integrate multiple issues, interactions and outcomes and then investigate trade-offs (Chen et al, 2012). Although not an equivalent example, the use of $\mathrm{BN}$ for environmental systems aligns with the outcomes of this paper as it can deal with multiple issues, interactions and outcomes. BN modelling offers several advantages, such as the ability to integrate qualitative and quantitative data, ease of communication due to transparent model structure, as well as the ability to handle data uncertainty (Pollino et al 2010 and Chaniak 1991). BN is also an adaptive modelling technique; the model structure and parameters can be updated as new data becomes available. The intended outcome of the $\mathrm{BN}$ is to develop an integrated understanding of the factors (i.e. individual, task and environment), while illuminating knowledge gaps and further areas that warrant investigation (Korb et al, 2010).

\section{PROBLEM FRAMING}

For most organisations, the scarcest and most expensive resource is their personnel. However, the benefits of managing this resource better are often overlooked (Bhaacharya, 2000). For high risk, physically demanding environments the pool of human resources decreases and the impacts of working in these conditions are detrimental to long term effectiveness of the personnel involved. The review of current literature has shown there is limited to no means of utilising basic health data to select personnel to operate in specific environments. Testing is limited to evaluating the health of the individual who is already operating in the environment (Harris et al, 2012), evaluating the effects of the environment (Maniscalco, 2010) or conducting a specific test to determine if someone meets the criteria (ADF, 1997). This research will develop a tool using 
BN to address this, with the solution utilising an individual's basic health data. Key considerations for problem framing are:

High Risk Environments. In a high-risk environment, the chance of getting killed or seriously injured is high, and there are many variables that cannot be controlled or mitigated, countering what is acceptable as normal day to day risk. The impacts of this environment are both physical and psychological.

Physically Demanding. SafeWork Australia defines a hazardous manual task, as defined in the WHS Regulations, as a task that requires a person to lift, lower, push, pull, carry or otherwise move, hold or restrain any person, animal or thing involving one or more of the following; repetitive or sustained force, high or sudden force, repetitive movement, sustained or awkward posture or exposure to vibration (Safework Australia, 2011).

What is required of an individual in a high risk physically demanding environment? The University of Western Australia defines selection criteria as representing the key qualifications, training, abilities, knowledge, personal attributes, skills and experience a person must have to do a job effectively, highlighting you must meet the selection criteria to be considered for a position (UWA, 2017). A high risk, physically demanding environment would increase the requirements of the selection criteria to only select personnel that can operate and succeed in this environment.

How do we measure what is required of the individual? Key outcomes of this process will be to determine how an individual is likely to react in the environment, wearing the equipment, doing the task that needs to be done and then being in an appropriate state to do it all again. In Amartya Sen's discussion about capability and well-being, she talks about the capability of a person reflecting the alternative combinations of functioning that the person can achieve (Sen, 1985). This is regarded as the capability approach used to evaluate an individual's ability to achieve various levels of functioning as part of living. General Healthcare Resources in the USA has solved this problem by defining the most strenuous and frequently performed tasks an individual is required to complete a specific role (US Department of Labour, 1991).

\section{MODEL CONCEPTUALISATION}

The process to develop an appropriate model begins with identifying the key elements or nodes. This includes: environmental impacts such as humidity, temperature and precipitation; and the capabilities of the individual, incorporating such aspects as gender, age and physiology, see Figure 1. The next step was to determine how to lay out the CM to represent all elements, without becoming too complex, and incorporating a structure that was conducive to becoming a BN (Korb et al, 2010). For this paper the CM has been broken into three sub-systems: physical environmental factors; individual related factors; and task related factors. By taking a systems view of the problem to represent the CM's concepts and relationships appropriately the three sub-systems allow it to be addressed in more manageable packages. The CM will use the relationship between the three sub-systems to determine the means of selection. As a comparison, Pollino et al (2010) utilise a risk assessment case study focusing on native fish communities in the Goulburn Catchment to further develop their methodology for parameterising and evaluating BN. The study develops a tool to undertake risk assessment of waterways within the Goulburn Catchment utilising BN, similar to what this study is trying to achieve with basic health data.

The initial structure of the BN will be developed from the three elements of the CM. Once the initial BN has been developed, the selected case study will be used to develop the network into an assessment tool to support the selection of individuals to operate in individual protective equipment (IPE) in a CBRNE environment. Here the outcome is to develop a metric for selecting the most appropriate personnel to operate within a CBRNE environment. This case study is a topical showcase as it will support a gap in the ability to select CBRNE operators to support military services to respond and operate at times where there are increasing threats of terrorist attacks. Findings from the study can be transferred to other applications, such as the selection of personnel to work in IPE for first responders, emergency and medical services.

In figure 1, we begin to see the nodes forming the CM focusing on either the environment they will be operating in, the traits we are looking for in the individual or the requirements that can be used to select the candidate. 
Guiding the development of the CM will be seeking to answer the following Critical Operational Issues (COI):

- What are the effects of the high-risk physically demanding environment?

- What are the requirements of an individual operating in a high risk, physically demanding environment?

- How do we measure what is required of the individual to operate in the environment?

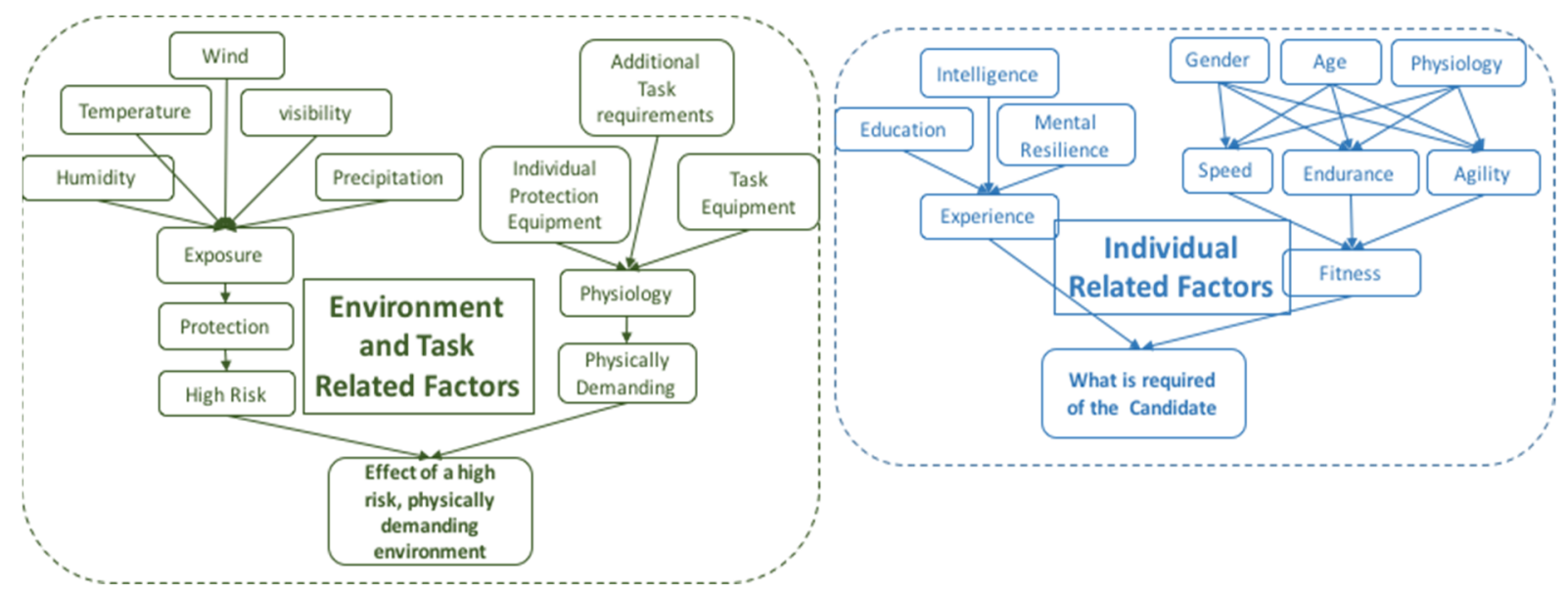

Figure 1. The preliminary CM

By addressing the BN solution through the physical environmental factors, individual related factors and task related factors to define the requirements we have defined the initial components of the model solution. To evolve the $\mathrm{CM}$ to a functional $\mathrm{BN}$ solution the nodes are allocated probability distribution functions, see figure 2. These probability distribution functions have been allocated by the author as illustrative data for this paper. While the initial relationship within each node is relatively binary, it is once the nodes begin to interact with the other nodes that true relationship effects must be defined. It is within these relationships that the impacts of one on the other must be prioritised, in this case physiology being rated over age, and then age higher than gender. This facilitates the tool to prioritise which will lead to the selection of one individual over another.

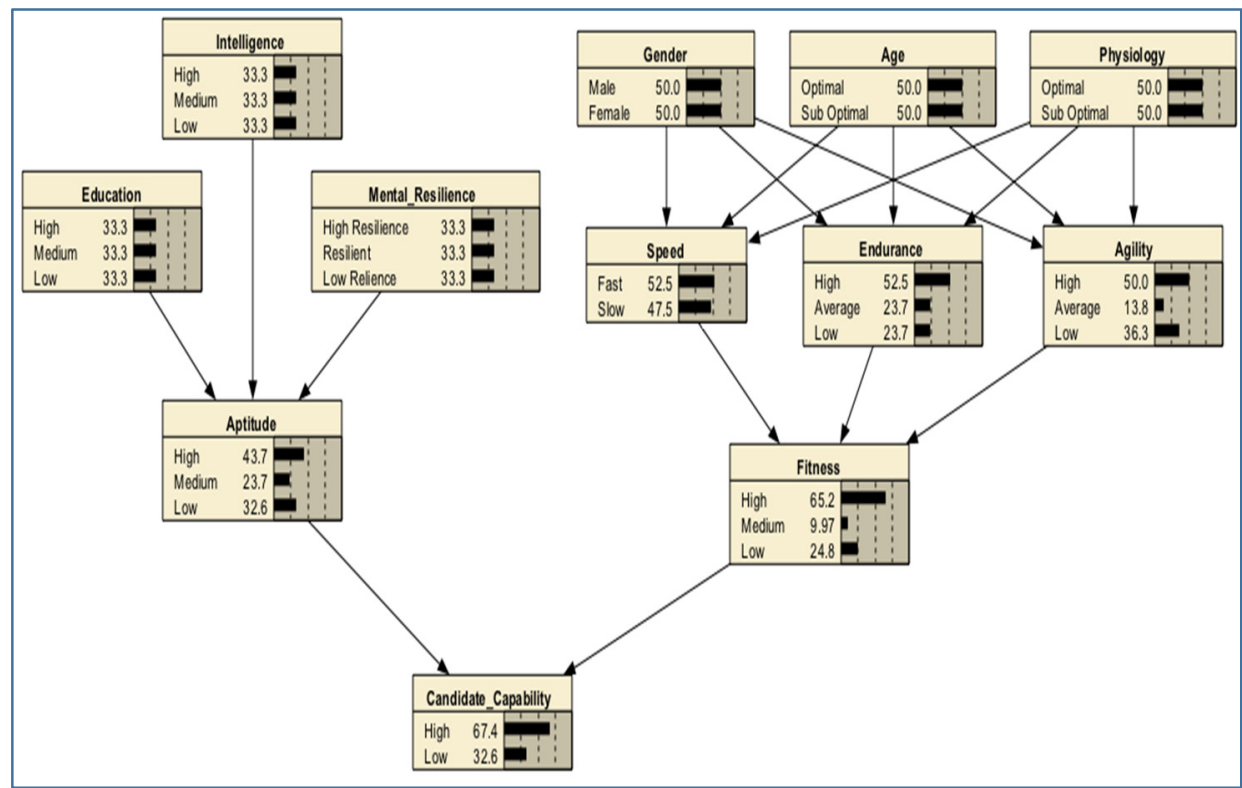

Figure 2. BN - Individual Related Factors

This process was repeated over the interactions between any parent and child nodes for the three subsystems. In figure 2, the interaction between humidity, temperature, wind, visibility and precipitation was too 
complex to be represented reasonably within a $\mathrm{BN}$, so the interactions were changed so that temperature and humidity fed into atmosphere, and then wind, visibility and precipitation feeds into external factors. These new interactions were then fed into exposure to simplify the BN.

\section{CASE STUDY}

The selected case study aims to develop an assessment tool to support the selection of individuals to operate in individual protective equipment (IPE) in a CBRNE environment. Here the intended outcome is to develop a metric for selecting the most appropriate personnel to operate within a CBRNE environment. The case study supports a gap in the ability to select CBRNE operators to support military and emergency services to operate more effectively if an incident occurred. The case study will evaluate the BN, and if we have selected the right individual based on previous experience, mental performance, etc, and they have the right aptitude, capable of meeting the expectations of the role and within the reasonable timeframes, then the requirements of the tool have been met.

\subsection{Exemplar Selection Process}

Using the developed BN, we have a range of inputs. For the individual inputs, these can be gained from an individual's personal file and form a data input requirement. The environmental inputs are what can be used to define the specific environments, either for more generic high risk physically demanding environments or, as an example, more specifically CBRNE environments. It is the changing of this requirement that will see the different individuals' strengths and weaknesses become obvious as the environments change. This happens as traits that are conducive to extreme environments facilitate the individual to be more effective than those without. In figure 3 this represents the final evolution of the BN process where the data has provided information on the capability and select-ability of the candidate. Figure 3 represents the inputs that can be provided and the $\mathrm{CM}$ is displaying how these inputs are interrelated to each other to form the final output, selection.

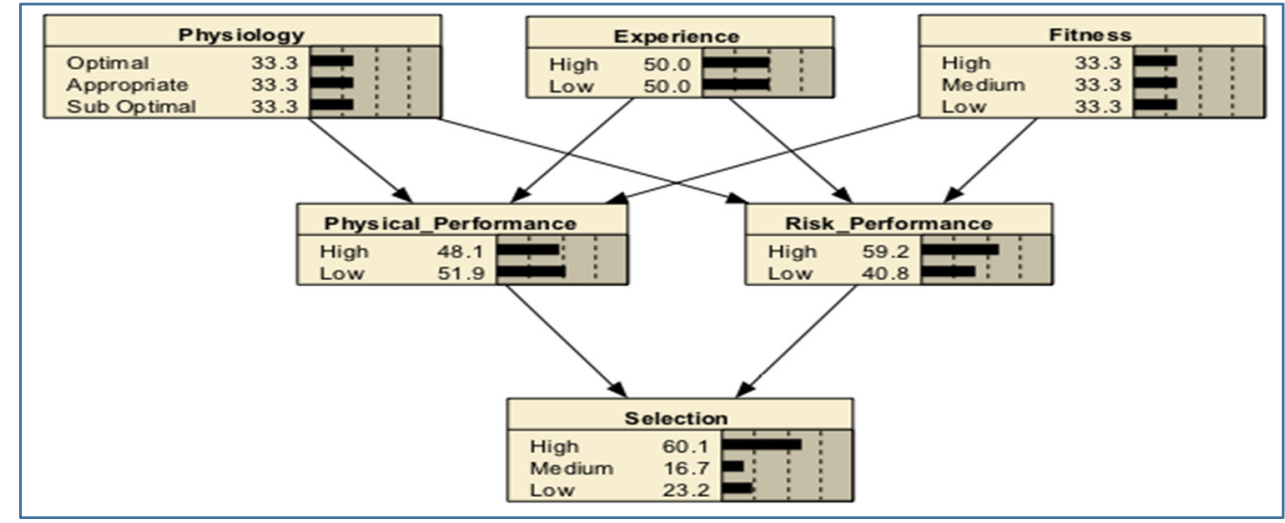

Figure 3. BN Requirements (Candidate 1)

Although the data collection process has begun, no actual individual information has been used, the following notional data was provided for the tool:

Candidate 1 - Average across all disciplines, we see $67.4 \%$ for individual capability, $68.2 \%$ for environmental capability and then a final selection score of $60.1 \%$. (See figure 3 )

Candidate 2 - Education - High, Intelligence - High, Mental Resilience - High etc, we see a 100\% appropriate candidate. (See figure 4)

Candidate 3 - This candidate is an older woman, but in all other aspects is high. Due to the weighting system, she is regarded as a $96.8 \%$ appropriate candidate. 


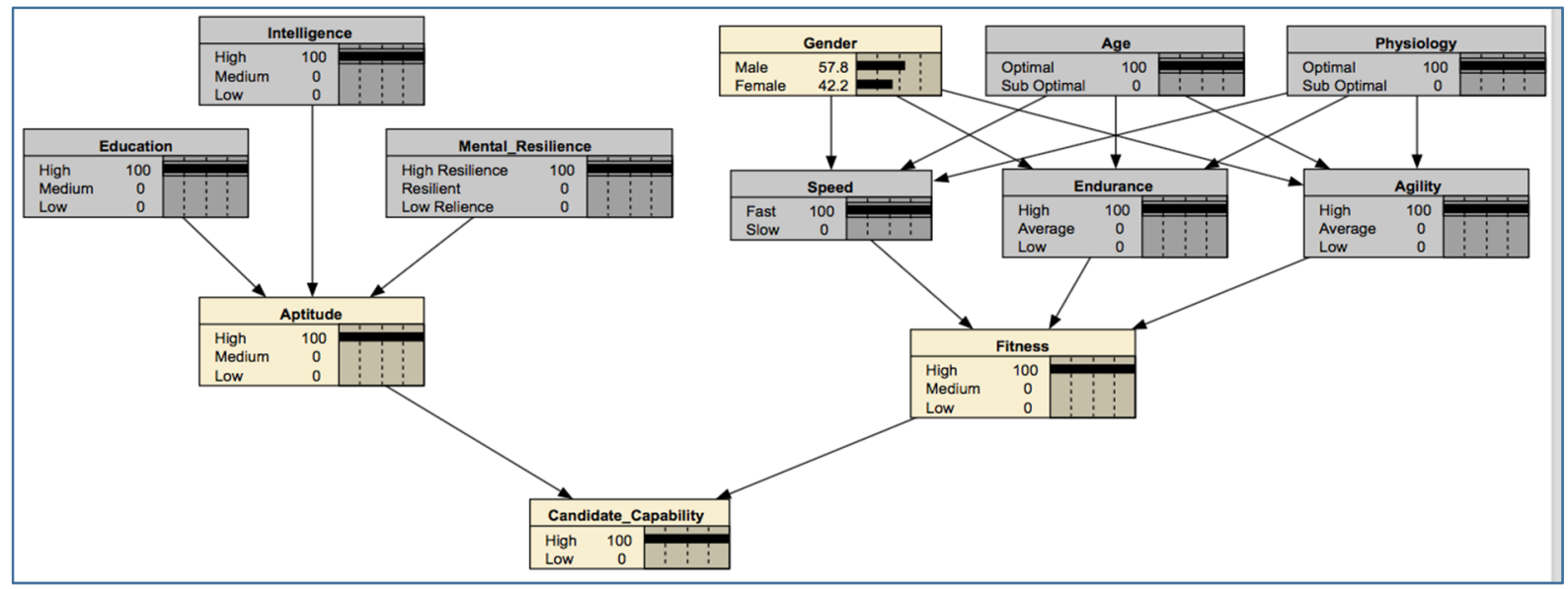

Figure 4. Candidate 2

All the BN have been populated to determine the interactions between the root inputs, which will eventually become the inputs for the selection mechanism. Currently, interactions have been determined by weighting certain attribute over others, for example, within figure 2; the three roots; gender, age and physiology have been weighted so that physiology is the most important trait, followed by age and then gender. For the intermediate level it is weighted endurance, agility, then speed. Where the initial values may be equally weighted, say $70 \%$, the second or third order trait would determine primacy. Looking at all figures, without inputting any data and accepting the even spread of data across the roots, we see that a candidate that is average, but across all traits, will be $67.4 \%$ suitable for the environment, over the next two steps of the BN, if they maintain their average results, they will be $60 \%$ suitable for selection. It will be up to the people selecting to determine the cut-off.

\section{DISCUSSIONS AND CONCLUSION}

The model has begun to identify: what the effects of the high-risk physically demanding environment are; what the requirements of an individual operating in a high risk, physically demanding environment are; and how we measure what is required of the individual to operate in the environment. Where the evolution from concept to $\mathrm{CM}$ has resulted in a $\mathrm{BN}$ solution, this solution is raw. To ensure a more effective $\mathrm{BN}$, the next phase is validation. This will confirm that in addition to identifying the most appropriate candidates, which will be achieved relatively easily, it will identify the individuals that meet the criteria but are not as easily identified. Probability distribution functions for the environmental factors need to explore the positive and negative effects, and as the tool currently distributes towards the good environment; long range visibility, low precipitation and low wind, it needs to be determined whether it is in fact counterproductive; and should be identifying the individual capable of better performing in the low visibility, high wind and high precipitation environment. By gaining data from personnel regarded as what the tool should be selecting, this data can be used to calibrate the weighting and data input requirements.

\section{REFERENCES}

Australian Defence Force (ADF) (1997). Publication 701 - Recruit Medical Examination Procedure. Canberra: ADF; 1997.

Arthur, J.B. (1994). Effects of human resource systems on manufacturing performance. Academy of Management Journal, 37(3): 670-687.

Bartel, A.P. (1994). Productivity gains from the implementation of employee training programs. Industrial Relations, 33(4): 411-425.

Becker, B. and Gerhart, B. (1996). The impact of human resource management on organisational performance: progress and prospects. Academy of Management Journal, 39: 779-801.

Bhaacharya, M. and Wright, P.M. (2000). Recognizing risk in human capital investments: A real options approach to strategic human resource management. CAHRS Working Paper 00-20, Cornell University, Center for Advanced Human Resource Studies.

Bhaacharya, M. \& Wright, P. M. (2000). Recognizing risk in human capital investments: A real options approach to strategic human resource management (CAHRS Working Paper \#00-20). Ithaca, NY: Cornell University, School of Industrial and Labor Relations, Center for Advanced Human Resource Studies. 
Mouthaan et al., A Conceptual Model for the Identification of Suitable Personnel to Operate in High-risk, Physically Demanding Environments

Charniak, E. (1991). Bayesian Networks without tears, AI Magazine, pp 50-64, winter.

Chen, S.H. and Pollino, C.A. (2012). Good Practice in Bayesian Networking, Environmental Modelling and Software, Vol 37, Nov, pp 134-145.

Harris, W.C., Ross, K.G., and Hancock, P.A. (2012). Changes in soldiers information processing under stress, contained within Performance Under Stress: Human Factors in Defence, edited by Szalma, J.R. and Hancock P.A., pp 101-104, Ashgate Publishing.

Hart, B.T. and Pollino, C.A. (2008) Increased use of Bayesian network models will improve ecological risk assessments, Human and Ecological Risk Assessment 14: 851-853.

Hart, B.T. and Pollino, C.A. (2009) Bayesian modelling for risk-based environmental water allocation. Canberra, National Water Commission.

Korb, K.B. and Nicholson, A.E. (2010). Bayesian Artificial Intelligence, $2^{\text {nd }}$ Edition, Chapman and Hall.

Lepak, D.P. and Snell, S.A. (1999). The human resource architecture: toward a theory of human capital allocation and development. Academy of Management Review, 24(1): 31-48.

Maniscalo, P.M., and Christen, H.T. (2010). Homeland Security: Principles and practices of terrorism response, Jones and Bartlett Publishers.

Pfeffer, J. (1994). Competitive advantage through people. California Management Review, 36(2): 9.

Physical demands as published by the US department of labor (1991) in the Dictionary of Occupational Titles, $4^{\text {th }}$, Edition.

Pollino, C.A., Woodberry, O., Nicholson, A., Korb, K. and Hart, B.T. (2007). Parameterisation and evaluation of Bayesian network for use in ecological risk assessment, Environmental Modelling and Software, Vol 22, Issue 8, Aug, pp 1140-1152.

SafeWork Australia (2011). Hazardous Manual Tasks, Code of Practice, Dec.

Sen, A. (1985) 'Capability and Well-being' within the text 'The quality of life', pp 30-49, Clarendon Press, Oxford.

University of Western Australia (UWA) (2017). http://www.jobs.uwa.edu.au/2180806

Woodberry, S. and Done, T. (2003). The use of Bayesian belief networks to aid in the understanding and management of large-scale coral bleaching; Townsville, Queensland. 